\title{
亚洲夏季风5400a BP极端减弱事件与文化演变
}

\author{
白益军 ${ }^{1}$, 张平 中 $^{1^{*}}$, 高涛 ${ }^{1}$, 余仁哲 ${ }^{1}$, 周鹏超 ${ }^{1}$, 程海 $^{2}$
}

1. 兰州大学地质科学与矿产资源学院, 甘肃省西部矿产资源重点实验室, 兰州 730000;

2. 西安交通大学全球环境变化研究院, 西安 710054

*通讯作者, E-mail: pzzhang@lzu.edu.cn

收稿日期: 2016-11-21; 接受日期: 2017-02-20; 网络版发表日期: 2017-03-20

国家自然科学基金项目(批准号: 41473009、41273014、40973007和40772110)资助

摘要 根据黄土高原西部万象洞石笋WXB07-4顶部 $22 \mathrm{~mm}$ 样品的平均误差仅约 $20 \mathrm{a}$ 年的 ${ }^{230} \mathrm{Th}$ 绝对定年数据和 平均时间分辨率为 $8 \mathrm{a}$ 的氧同位素数据, 建立了亚洲季风边缘区中晚全新世过渡期亚洲夏季风变迁的时间序列. 结果发现, 从6420到4920a BP期间, 亚洲夏季风呈现出逐渐减弱的趋势, 并以 6420 6170a BP之间的季风强盛期 和较高降水量、6170 5700a BP期间的夏季风平稳波动和在5700 4920a BP期间夏季风出现极端减弱期为主要 特征. 特别是以 5430a BP 为突变年代出现在5700 4920a BP期间的亚洲夏季风特征极端减弱事件(5400a或5.4ka 事件), 经历了约 $290 \mathrm{a}$ 的减弱阶段和约350a的增强阶段, 在定年误差范围之内与亚洲季风区其他地区洞穴石笋记 录具有等时性. 通过与过去7000a考古资料的对比发现, 中国黄河流域仰韶文化和西辽河流域红山文化早期的 衰落发生在夏季风降水由多到少的变化期; 5400a BP极端夏季风减弱事件发生造成的降水大幅度减少可能与 5500a BP左右黄河流域渭河河谷关中地区杨官寨遗址庙底沟文化、河南西部仰韶中期文化和下游大汶口早期 文化、辽河河谷西部红山中期文化的衰落存在一定程度上的联系; 5400a BP事件后期 (5430 4920a BP) 与亚洲 夏季风相联系的降水小幅度逐渐增加和随后的剧烈减少可能也与仰韶晚期文化和红山晚期文化的共同繁荣和 消失、渭河关中盆地以杨官寨遗址为代表的仰韶晚期文化和上游地区大地湾四期仰韶晚期文化的消失、大汶 口中期文化的开始与晚期文化的形成以及龙山文化的兴起、长江中下游崛起的屈家岭文化和良渚文化的相联 系。通过与高原石笋降水记录对比发现, 高海拔地区夏季风降水在 $5400 \mathrm{a} \mathrm{BP}$ 事件后期的增加比在低海拔地区显 著并具有更大幅度的波动, 可能与 $5300 \mathrm{a} \mathrm{BP}$ 左右马家窑文化在黄河上游河谷地带的兴起及其扩张有关.

关键词亚洲夏季风, 5400a BP极端减弱事件, 新石器晚期文化演变, 万象洞石笋氧同位素, 西黄土高原

\section{1 引言}

作为全球季风系统最重要的子系统, 亚洲季风 以其降水多变的特点影响着全球大约 $1 / 2$ 人类的生存 和发展(Webster等, 1998; Mohtadi等, 2016). 亚洲季风
区洞穴石笋以其高精度 ${ }^{230} \mathrm{Th}$ 定年和高时间分辨率揭 示了过去 $640 \mathrm{ka}$ 轨道和千年尺度上的季风演变规律 (Wang 等, 2001, 2008; Yuan等, 2004; Cheng等, 2009, 2012, 2016; Kathayat等, 2016). 而对于全新世间冰 期北高纬地区发生的 8 个世纪尺度上的剧烈降温事

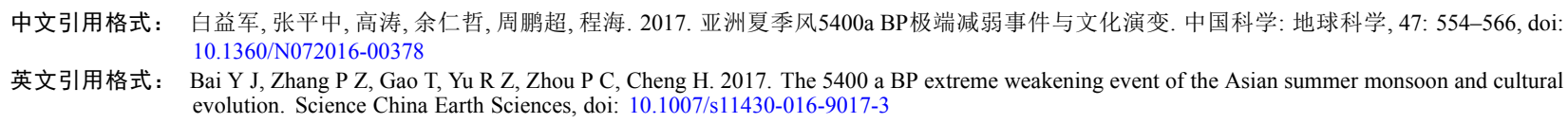
10.1360/N072016-00378

英文引用格式： Bai Y J, Zhang P Z, Gao T, Yu R Z, Zhou P C, Cheng H. 2017. The 5400 a BP extreme weakening event of the Asian summer monsoon and cultural evolution. Science China Earth Sciences, doi: 10.1007/s11430-016-9017-3 
件(Wanner等, 2008, 2011)在亚洲季风区的研究仅仅 局限于 $9200 \mathrm{a}(9.2 \mathrm{ka})$ 和 $8200 \mathrm{a}(8.2 \mathrm{ka})$ 季风极端减弱事件 (Cheng等, 2009; Dykoski等, 2005; Fleitmann等, 2008; Wu等, 2012; Liu等, 2013; Liu和 Hu, 2016) 以及早期农 业社会的响应(Flohr等, 2016). 基于亚洲季风区不同石 笋年代误差和分辨率差异建立的最新非线性数据网 络分析(Donges等, 2015)表明, 亚洲季风区确实存在与 全新世快速气候变化和北高纬Bond事件相匹配的、

分别在8.5 7.9、7.3、5.7 5.0、4.1 3.7和3.0 2.4ka BP 出现的极端减弱事件, 而且这些极端事件明显地与史 前文化的发生、扩张和崩溃有关.

亚洲季风区过去气候变化档案, 如中国黄土序列 (An等, 2004)、印度湖泊记录(例如, Dixit等, 2014)、石 笋记录(Wang等, 2005; Berkelhammer等, 2012)揭示出 的 $4200 \mathrm{a}$ 极端干早事件导致新石器人类文化和印度河 谷西北部Harappan城市文明的崩溃(Wu和Liu, 2004), 而 且这个极端降温事件也可能造成北半球中纬埃及古王 国、希腊早期青铜器时代和亚洲西南部美索不达米亚 Akkadian古老帝国遭遇的持续干早直至崩溃(Cullen等, 2000; Marshall等, 2011; Welc和Marks, 2014). 但是对于 在中全新世高温期/新冰期转换期间发生在 6000 5000 a BP的极端气候突变事件(Wanner等, 2008; Kaufman 等, 2016), 虽然在亚洲季风区的黄土沉积(Huang等, 2000; Maher和Hu, 2006; Maher, 2008) 和低纬度几个湖 泊(Mischke和Zhang, 2010; An等, 2012; Dixit等, 2014; Prasad等, 2014)、现代夏季风边界附近中纬度湖泊 (Xiao等, 2009)和亚洲季风区石笋数据的再分析(Donges 等, 2015)中均有体现, 并且作为重要的快速气候变化事 件来阐述新石器文化与气候的相互作用(杨颖, 2013)、 早期普通粟类和稻类农业的向西传播与小麦和大麦 农业的向东扩散(Li等, 2007; Li等, 2011; Jia等, 2013; Dodson等, 2013; Barton和An, 2014; 李小强, 2013). 同 时这个时期发生的极端温度降低和降水减少事件可 能直接导致欧洲早期农业社会的崩溃(Budja, 2015)、 幼发拉底河中上游的美索不达米亚乌鲁克文化的扩 张(Clarke等, 2016)、撒哈拉东部史前人类在 5300a BP 左右向埃及尼罗河谷的迁徙和印度西北部Harappan文 明的城市化(Prasad等, 2014)等. 当然我们不能排除一 些不具普遍性的差异性社会因素如战争、疾病和宗 教活动等(Butzer和Endfield, 2012)、偶尔发生的其他 自然灾害如地震(Wu等, 2016)对史前文化的作用. 然
而, 由于所选取的环境载体及其定年精度和时间分辨 率的限制, 对于事件的精确年代、持续时间和变化模 式以及变化幅度等都不能进行详细的描述, 导致气候 变化事件与文化程度的非等时性, 限制了我们理解这 种世纪-亚世纪尺度的季风减弱事件的动力学和对史 前人类文化的作用.

鉴于精确定年和高分辨率洞穴石笋能够揭示亚 洲夏季风降水减少造成中国历史社会动荡和朝代更 迭(Zhang等, 2008; Tan等, 2011, 2015)的优势. 本文以 黄土高原西部甘肃万象洞石笋WXB07-4顶部 $22 \mathrm{~mm}$ 纹 层清晰且致密无沉积间断的部分作为研究对象, 通 过高精度 ${ }^{230} \mathrm{Th}$ 绝对定年和高分辨率氧同位素数据测 定, 建立误差大约 $20 \mathrm{a}$ 和平均分辨率为 $8 \mathrm{a}$ 的亚洲夏季 风5400a (5.4ka BP)极端减弱事件变化的时间序列, 确 定事件的起止时间、持续时间和变化模式. 通过对比 亚洲季风区其他典型石笋记录, 探讨石笋记录的亚洲 季风演变的降水区域差异. 同时, 结合考古资料, 论述 我国新石器文化的发生、发展和迁徙; 以及通过与青 藏高原高海拔石笋记录的对比, 评估新石器文化向青 藏高原扩张的原因, 揭示亚洲夏季风中晚全新世过渡 时期亚洲夏季风极端突变的特征与新石器文化发生 发展的联系.

\section{2 样品与实验}

万象洞 $\left(33^{\circ} 19^{\prime} \mathrm{N}, 105^{\circ} 00^{\prime} \mathrm{E}, 1200 \mathrm{~m}\right.$ asl; 图1)(Zhang 等, 2004, 2008; Johnson等, 2006)位于现代亚洲季风与 西风急流相互作用(Schiemann等, 2009; Dallmeyer等, 2013; Chiang等, 2015)最敏感地区的黄土高原西部和 青藏高原东部、现代亚洲夏季风的边缘区域或前端 地区, 对亚洲夏季风的变化相应非常敏感. 而且, 这个 地区邻近被称为古老中华文明摇篮黄河流域的渭河关中地区(张之恒, 2009).

洞穴所在地甘肃武都地区年均温为 $12.6^{\circ} \mathrm{C}$, 年均 降水量 $489 \mathrm{~mm}, \sim 80 \%$ 以上的降水集中在夏季风盛行的 5 9月, 属于典型的季风性半干旱气候. 该洞目前已经 开发的长度约 $1400 \mathrm{~m}$, 发育在石炭纪灰岩中, 洞穴顶部 覆盖有30 250m 厚的石灰岩和约 $10 \mathrm{~m}$ 厚的黄土, 上覆 植被稠密. 洞内现代次生碳酸盐发育且有大量现代沉 积, 洞穴内温度基本恒定在 $11^{\circ} \mathrm{C}(2001$ 年 8 月至 2003 年 1月), 相对湿度达 $100 \%$, 沉积环境较为稳定, 为弱通风 


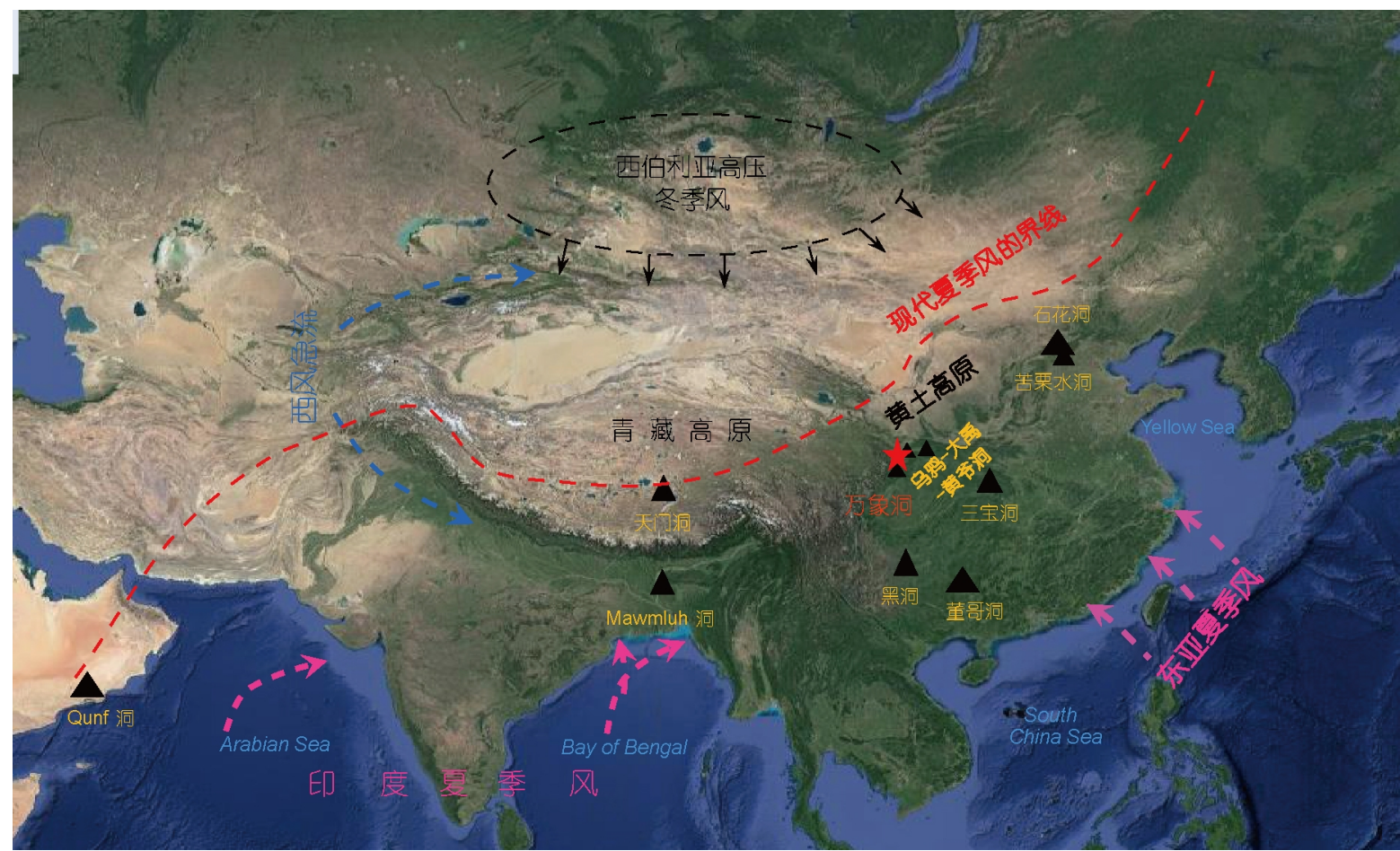

图 1 万象洞(红色五角星)在亚洲季风区的位置图

根据Google Earth制作. 在图中画出了现代亚洲夏季风大约界线、印度夏季风和东亚夏季风的方向、西伯利亚高压冬季风和西风急流的大约 位置. 在本文中对比和引用的几个洞穴位置也用黑色三角形标记在图中, 其中包括中国西藏天门洞 $\left(30^{\circ} 55^{\prime} \mathrm{N}, 90^{\circ} 40^{\prime} \mathrm{E}, 4800 \mathrm{~m}\right.$ asl; Cai等, 2012)、 湖北三宝洞 $\left(31^{\circ} 40^{\prime} \mathrm{N}, 110^{\circ} 26^{\prime} \mathrm{E}, 1900 \mathrm{~m}\right.$ asl; Dong等, 2010; Wang等, 2008; Cheng等, 2012, 2016)、贵州董哥洞 $\left(25^{\circ} 17^{\prime} \mathrm{N}, 108^{\circ} 5^{\prime} \mathrm{E}, 680 \mathrm{~m}\right.$ asl; Yuan

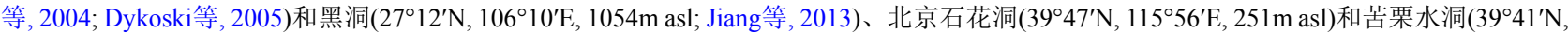

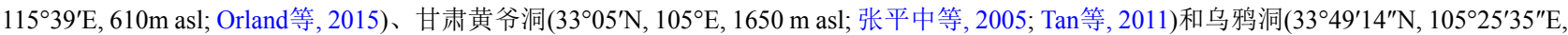
$1370 \mathrm{~m}$ asl; Tan等, 2014)、陕西大禹洞 $\left(33^{\circ} 08^{\prime} \mathrm{N}, 106^{\circ} 18^{\prime} \mathrm{E}, 870 \mathrm{~m}\right.$ asl; Tan等, 2015), 印度东北部的Mawmluh洞 $\left(25^{\circ} 15^{\prime} 44^{\prime \prime} \mathrm{N}, 91^{\circ} 52^{\prime} 54^{\prime \prime} \mathrm{E}, 1290 \mathrm{~m}\right.$ asl; Berkelhammer等, 2012)和阿曼的Qunf洞 $\left(17^{\circ} 10^{\prime} \mathrm{N}, 54^{\circ} 18^{\prime} \mathrm{E}\right.$; 650m asl; Fleitmann等, 2003, 2007).

洞穴(Zhang等, 2004, 2008; Johnson等, 2006). 2007年6 月, 万象洞管理部门在洞口附近清理堆积物时, 意外 在洞口附近发现了另一个一直封闭的洞穴(万象新洞). 万象新洞洞穴平均温度比万象洞高约 $1.3^{\circ} \mathrm{C}$ (桑文翠等, 2013), 并且内部出现大量化石石笋、现代洞穴滴水和 现代石笋沉积物, 保留大量的洞穴边池池水.

本文研究的石笋样品WXB07-4于 2007年6月采自 万象洞新洞. 在实验室沿生长轴的切面抛光后呈白 色-淡黄色, 具有清晰的纹层, 纯净致密且无沉积间断 (图2a). 使用牙钻和刻刀相结合的方法沿垂直于石笋 生长轴且平行于生长纹层进行室内子样品采集(Yang 等, 2007; Zhang等, 2010). WXB07-4石笋顶部22mm共 刮取 370 个同位素子样品, 为了防止样品之间的交叉 混染, 我们采用间隔挑样方法选取 185 个子样品进行 稳定氧同位素测试. 测试工作在中国科学院南京地质
古生物研究所同位素实验室完成. 每 10 个样品内插一 个标准样品, 结果以 $\mathrm{PDB}$ 标准给出, 用符号 $\delta$ 表示, $\delta=$ $\left[\left(R_{\text {样品 }} / R_{\text {标准 }}\right)-1\right] \times 1000$, 其中 $R={ }^{18} \mathrm{O} /{ }^{16} \mathrm{O}$, 分析误差 $( \pm 2 \sigma)$ 优于 $0.05 \%$.

绝对 ${ }^{230} \mathrm{Th}$ 定年样品用直径 $0.5 \mathrm{~mm}$ 的牙钻在生长轴 附近平行于纹层钻取。测试工作在美国Minnesota大 学地质与地球物理系地质年代学实验室完成, 测试 方法见文献(Shen等, 2002; Cheng等, 2000, 2013), 分析 仪器为MC-ICP-MS(Neptune), 年龄误差为 $\pm 2 \sigma$ 测量统 计误差。

\section{3 结果与讨论}

\section{1 石笋WXB07-4顶部 $22 \mathrm{~mm}$ 的 ${ }^{230} \mathrm{Th}$ 年代模型} 石笋WXB07-4顶部的 8 个 ${ }^{230} \mathrm{Th}$ 年代数据见表 1 . 
表 1 万象洞石笋WXB07-4的MC-ICP-MS铀系测年结果 ${ }^{\text {a) }}$

\begin{tabular}{|c|c|c|c|c|c|c|c|c|}
\hline 样品编号 & $\begin{array}{l}\text { 距顶距 } \\
\text { 离 }(\mathrm{mm})\end{array}$ & $\begin{array}{c}{ }^{238} \mathrm{U}\left(\times 10^{-6}\right) \\
\left(\mathrm{g} \mathrm{g}^{-1}\right)\end{array}$ & $\begin{array}{c}{ }^{232} \mathrm{Th}\left(\times 10^{-12}\right) \\
\left(\mathrm{g} \mathrm{g}^{-1}\right)\end{array}$ & $\begin{array}{c}\delta^{234} \mathrm{U} \\
\text { 测量值 }\end{array}$ & $\begin{array}{c}{ }^{230} \mathrm{Th} /{ }^{238} \mathrm{U} \\
\text { 活度比 }\end{array}$ & $\begin{array}{l}{ }^{230} \mathrm{Th} \text { 年龄 } \\
\text { (a, 未校正) }\end{array}$ & $\begin{array}{c}\delta^{234} U_{\text {初始值 }} \\
\text { 校正值 }\end{array}$ & $\begin{array}{c}{ }^{230} \mathrm{Th} \text { 年龄 } \\
(\mathrm{a} \mathrm{BP}, \\
\text { 校正年龄) }\end{array}$ \\
\hline WXB074-1 & 1.0 & $1.068 \pm 1$ & $438 \pm 10$ & $1082.7 \pm 2.7$ & $0.0972 \pm 0.0004$ & $5194 \pm 23$ & $1099 \pm 3$ & $5128 \pm 24$ \\
\hline WXB074-2.5 & 2.5 & $1.236 \pm 1$ & $80 \pm 6$ & $1198.0 \pm 2.4$ & $0.1089 \pm 0.0005$ & $5518 \pm 29$ & $1217 \pm 2$ & $5454 \pm 29$ \\
\hline WXB074-2 & 4.0 & $1.913 \pm 2$ & $1337 \pm 28$ & $1173.4 \pm 2.8$ & $0.1113 \pm 0.0004$ & $5709 \pm 23$ & $1192 \pm 3$ & $5640 \pm 24$ \\
\hline WXB074-7 & 7.0 & $1.300 \pm 1$ & $438 \pm 11$ & $1139.1 \pm 2.2$ & $0.1153 \pm 0.0005$ & $6012 \pm 25$ & $1159 \pm 3$ & $5944 \pm 25$ \\
\hline WXB074-3 & 10.5 & $1.344 \pm 2$ & $384 \pm 9$ & $1129.0 \pm 2.6$ & $0.1189 \pm 0.0003$ & $6239 \pm 18$ & $1149 \pm 3$ & $6176 \pm 18$ \\
\hline WXB074-4 & 14.0 & $1.440 \pm 2$ & $1208 \pm 25$ & $1107.5 \pm 2.7$ & $0.1191 \pm 0.0003$ & $6314 \pm 20$ & $1127 \pm 3$ & $6242 \pm 22$ \\
\hline WXB074-5 & 17.5 & $1.354 \pm 1$ & $756 \pm 16$ & $1138.4 \pm 2.8$ & $0.1232 \pm 0.0004$ & $6438 \pm 23$ & $1159 \pm 3$ & $6371 \pm 24$ \\
\hline WXB074-6 & 22 & $1.483 \pm 2$ & $2540 \pm 51$ & $1133.9 \pm 2.8$ & $0.1241 \pm 0.0004$ & $6503 \pm 22$ & $1155 \pm 3$ & $6420 \pm 27$ \\
\hline
\end{tabular}

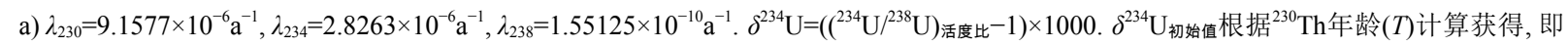
$\delta^{234} \mathrm{U}_{\text {初始值 }}=\delta^{234}$ 测量值 $\times \mathrm{e}^{\lambda 234} \times T,{ }^{230} \mathrm{Th}$ 年龄的校正值是假设初始 ${ }^{230} \mathrm{Th} /{ }^{232} \mathrm{Th}$ 原子比为 $(4.4 \pm 2.2) \times 10^{-6}, \mathrm{a} \mathrm{BP}=$ 距目前 $(1950 \mathrm{AD})$ 的年代

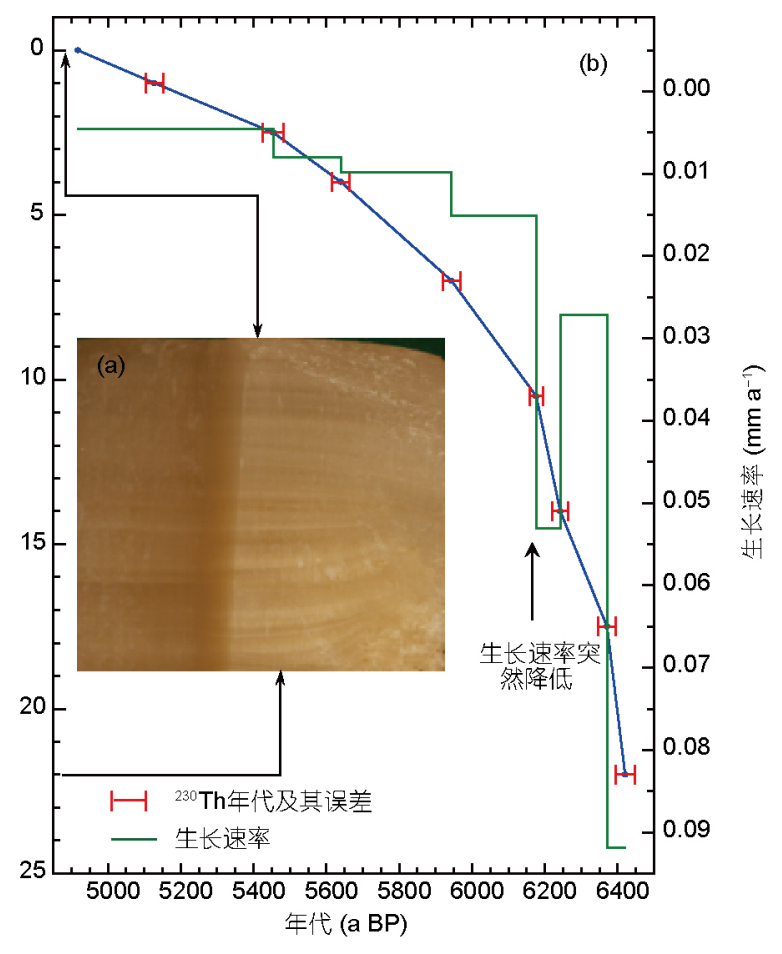

图 2 石笋WXB074顶部22mm剖面图(a)及其年代模型 (蓝色)和生长速率分布图(b)

石笋样品的 ${ }^{238} \mathrm{U}$ 含量高, 在 $1.068 \times 10^{-6} \sim 1.913 \times 10^{-6} \mathrm{~g} \mathrm{~g}^{-1}$, 平 均值为 $1.39 \times 10^{-6} \mathrm{~g} \mathrm{~g}^{-1}$; 而碎屑 ${ }^{232} \mathrm{Th}$ 含量则很低 $\left(0.08 \times 10^{-9} \sim 2.5 \times 10^{-9} \mathrm{~g} \mathrm{~g}^{-1}\right.$, 平均值为 $\left.0.898 \times 10^{-9} \mathrm{~g} \mathrm{~g}^{-1}\right)$, 所 以我们能够获得误差在 $\pm 18 \sim \pm 29 \mathrm{a}$ 、平均误差为 $24 \mathrm{a}$ 的高精度 ${ }^{230} \mathrm{Th}$ 定年数据.

我们采用相邻年代点线性内插法建立石笋
WXB07-4顶部 $22 \mathrm{~mm}$ 的年代模型(图 $2 \mathrm{~b}$ ). 从图 $2 \mathrm{~b}$ 中可 以看出石笋从 $6420 \mathrm{a} \mathrm{BP}$ 开始到 $4920 \mathrm{a}$ BP $(0 \mathrm{~mm})$ 停止生 长的 $1500 \mathrm{a}$ 期间, 生长(沉积)速率逐渐减小, 从 0.092 减 小到 $0.005 \mathrm{~mm} \mathrm{a}^{-1}$; 其中在 $6180 \mathrm{a} \mathrm{BP}$ 左右沉积速率突然 发生大幅度变化, 从前期平均值 $0.057 \mathrm{~mm} \mathrm{a}^{-1}$ 突然减小 至后期平均值的 $0.009 \mathrm{~mm} \mathrm{a}^{-1}$, 减小幅度达到 6.4 倍, 这 两种沉积速率变化状态可能反映了石笋生长条件的 大幅度变化.

\section{2 石 笋 WXB07-4 顶 部 在 6420 4920a BP 期 间 $\delta^{18} \mathrm{O}$ 的时间序列}

石笋WXB07-4顶部在 6420 4920a BP 期间 $\delta^{18} \mathrm{O}$ 值 的变化范围在 $-11.25 \sim-8.10 \%$, 平均时间分辨率为 $8 \mathrm{a}$, 变化幅度达到 $3.15 \%$ (图3a).

近20年亚洲季风区众多洞穴记录研究表明,石笋氧 同位素组成无论是在冰期/间冰期和千年还是百年/十 年等时间尺度上, 都与指示夏季风强度变化的夏/冬 降水比率、热带水汽源与洞穴所在地的距离(即, 源 区远近)和地方性的降水量有关(Yuan等, 2004; Wang 等, 2005, 2008; Cheng等, 2012, 2016; Dykoski等, 2005; Fleitmann等, 2003, 2007; Zhang等, 2008; Dong等, 2010; Berkelhammer等, 2012; Jiang等, 2013), 换句话说石笋 $\delta^{18} \mathrm{O}$ 是以上几种因素的集成或是季风降水总强度的反 映(Cheng等, 2016), 即季风区中某一具体区域的降水 量与石笋 $\delta^{18} \mathrm{O}$ 值之间没有简单的线性关系, 这种现象 在中国东南部不同洞穴石笋 $\delta^{18} \mathrm{O}$ 记录中表现比较明显 


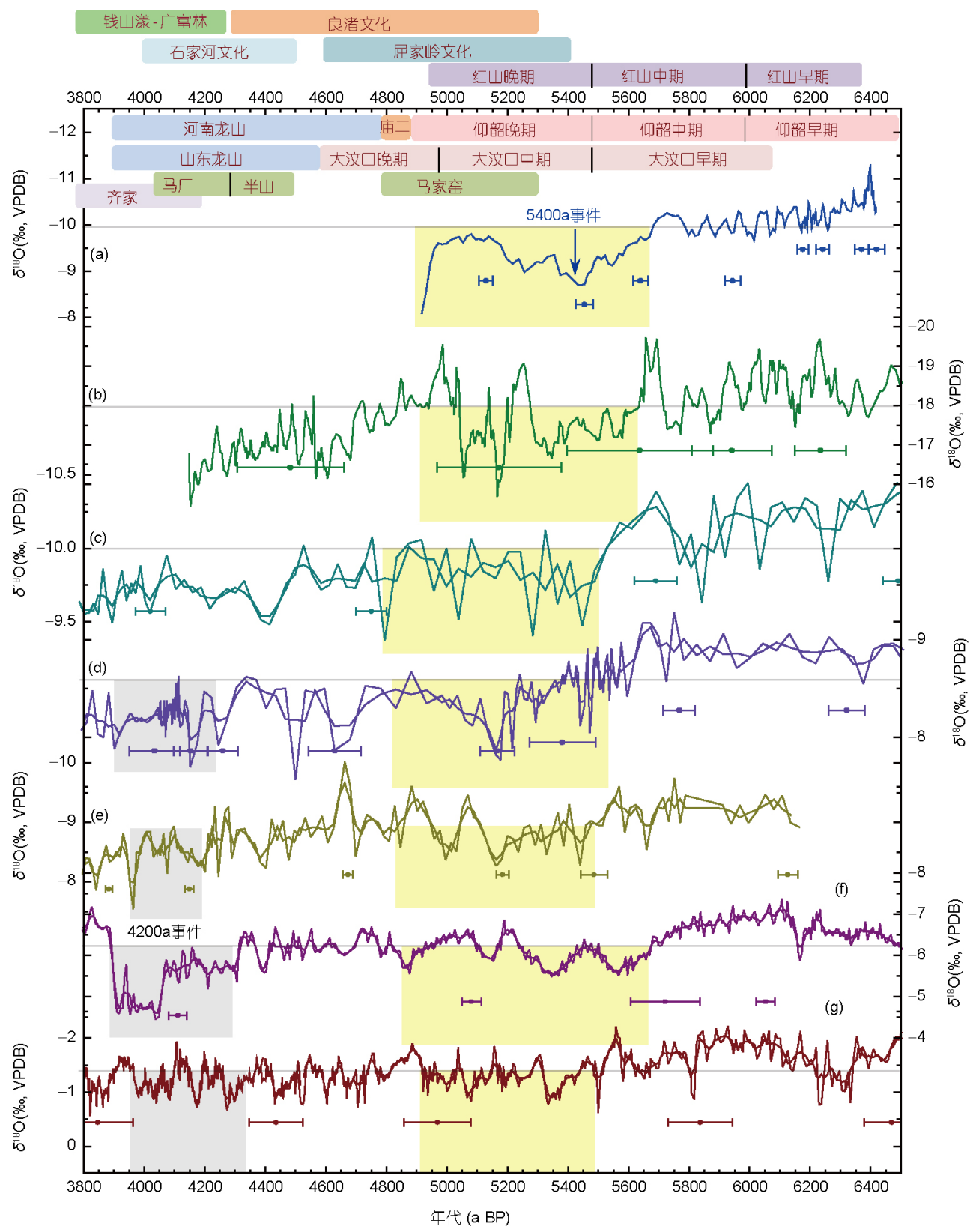

图 3 万象洞WXB07-4石笋顶部 $\delta^{18} \mathbf{O}$ 时间序列与亚洲季风区部分洞穴石笋记录的对比

(a) 中国万象洞WXB07-4顶部石笋 $\delta^{18} \mathrm{O}$ 记录(蓝色, 本文); (b) 中国青藏高原天门洞石笋TM-18 $\delta^{18} \mathrm{O}$ 记录(橄榄绿色; Cai等, 2012); (c) 中国三宝 洞石笋SB43 $\delta^{18} \mathrm{O}$ 记录(深青绿色; Dong等, 2010); (d) 中国贵州董哥洞D4石笋 $\delta^{18} \mathrm{O}$ 记录(紫罗兰色; Dykoski等, 2005); (e). 中国贵州黑洞D1和D2 石笋 $\delta^{18} \mathrm{O}$ 记录(深黄色; Jiang等, 2013); (f) 印度东北部Mawmluh洞KM-A石笋 $\delta^{18} \mathrm{O}$ 记录(紫色; Berkelhammer等, 2012); (g) 阿曼Qunf洞Q5石笋 $\delta^{18} \mathrm{O}$ 记录(酒红色; Fleitmann等, 2003, 2007). 图3(c) (e)中的粗线是 3 点滑动, 而(f)和 $(\mathrm{g})$ 中的粗线是 5 点滑动; 黄色阴影指示 $5400 \mathrm{a} \mathrm{BP}$ 事件的时 代范围; 灰色阴影指示了 4200a BP事件的可能时代范围(Berkelhammer等, 2012); 横的灰色线指示与万象洞同时期 6420 4920a BP的氧同位素 平均值, 其中WXB $074=-10.01 \%$ 、 TM $18=-17.99 \%$ 、 SB $43=-10.03 \%$ 、 D $4=-8.60 \%$ 、 KM-A $=-6.38 \% 0$ 和 $\mathrm{Q} 5=-1.50 \%$, 黑洞由于数据年代未到 6420a BP, 没有计算平均值; 图内的最上部标出了仰韶文化(Liu和Chen, 2012; Barton等, 2009)、红山文化(孙妙凝, 2016)、马家窑文化和齐家 文化(Dong等, 2013)、庙二文化(韩建业, 2006)、河南/山东龙山文化(Liu和Chen, 2012; 王守功, 2004)、大汶口文化-良渚文化-屈家岭文化和 石家河文化(Liu和Chen, 2012; Jin等, 2016)、钱山漾和广富林文化(http://www.zj.xinhuanet.com/newscenter/focus/2014-11/16/c_1113266903.htm; 许鹏飞, 2015)的时代范围和划分 
(例如, Cheng等, 2012). 但对处在亚洲夏季风前端或者 边缘地区的、以具有明显季节性并以夏季风降水(降 水 $\delta^{18} \mathrm{O}$ 值相对偏负)为主的中国北部和西北部季风区内 降水多少与夏季风强弱的关系则相对简单, 基本上显 示正相关关系(Baker等, 2015; Duan等, 2016). 甘肃万 象洞(Zhang等, 2008)及其附近的黄爷洞(Tan等, 2011) 和乌鸦洞(Tan等, 2014)、陕西大禹洞(Tan等, 2015) 以 及北京石花洞(李向否个人交流)中的石笋 $\delta^{18} \mathrm{O}$ 记录研 究均证明, 偏负的石笋 $\delta^{18} \mathrm{O}$ 值代表与夏季风增强有关 的夏季降雨量增加相对应, 反之亦然. 而且, 这些记录 和现代器测资料分析(Ding等, 2008)、季节分辨率的 苦栗水洞石笋氧同位素分析(Orland等, 2015)和气候模 拟结果(Liu等, 2014)相一致.

石笋WXB07-4顶部的 $\delta^{18} \mathrm{O}$ 值时间序列显示出从 6420a BP开始到4920a BP期间的逐渐变得偏重和沉积 速率逐渐减小趋势(图 $2 b$ 和 $3 a$ ), 指示了亚洲夏季风强 度的逐渐减弱和与之有联系的降水量减少.

万象洞 $\delta^{18} \mathrm{O}$ 逐渐偏重的长趋势显示出 3 相特征(图 $3 \mathrm{a})$ : 在第 1 相的 6420 6170a BP期间, $\delta^{18} \mathrm{O}$ 值显示出以 波动幅度为 $1.38 \%$ 的、在 $-11.25 \sim-9.87 \%$ (平均值为 $-10.41 \%$ ) 波动的逐渐偏重趋势, 而且平均沉积速率高 达 $0.057 \mathrm{~mm} \mathrm{a}^{-1}$ (图 $1 \mathrm{~b}$ )和子样品同位素数据时间分辨率 达到 $3 \mathrm{a}$, 说明这期间具有较高的降水量, 与较强的夏 季风有关; 而在6170 5700a BP期间的第2相(图3a), 沉 积速率突然下降到 $0.012 \mathrm{~mm} \mathrm{a}^{-1}$ (图2b), 数据时间分辨 率降低到 $11 \mathrm{a}$, 并且 $\delta^{18} \mathrm{O}$ 以 $-10.39 \sim-9.67 \% \mathrm{o}$ 的小幅度波 动(幅度仅 $0.72 \%$ )、平均值 $-10.05 \%$ 几乎等于整体平 均值为特征, 是夏季风突然变化到平稳波动的反映. 最有趣的是 5700 4920a BP期间的第3相, $\delta^{18} \mathrm{O}$ 值波动 在-9.99 - $8.10 \%$ (波动幅度高达 $1.89 \%$ ), 平均值仅为 $-9.35 \%$, 均大于整体平均值 (图3a), 同时沉积速率出 现几乎达到 1 倍的、与降水量大幅度再次下降有关的 大幅度降低期(图2b), 时间分辨率也降低到最低(15a), 指示了夏季风的大幅度极端变化. 同时, 在第 3 相期 间(图3a), 以 5430a BP处的 $\delta^{18} \mathrm{O}$ 值 $-8.70 \%$ 极端偏重信 号为主的亚洲夏季风极端减弱谷, 显示出从第2相末 期的 5730a BP开始到 5430a BP的逐渐偏正和从 5430a BP到 5080a BP逐渐偏负的波动趋势, 指示亚洲夏季风 以 5430a BP为突变点的 5400a 极端减弱时期内 (5400a 或 $5.4 \mathrm{ka}$ 事件)夏季风经历了 $290 \mathrm{a}$ 的逐渐减弱然后经历 $350 \mathrm{a}$ 的逐渐增强总体特征.

\section{3 万象石笋WXB07-4 顶部的 $\delta^{18} \mathrm{O}$ 记录与亚洲季 风区其他石笋记录的对比}

为了揭示高精度定年和高分辨率万象石笋 WXB07-4 $\delta^{18} \mathrm{O}$ 记录的亚洲夏季风强度变化的区域模 式, 我们把这期间具有大幅度波动的、定年精度和分 辨率相对较高的中国青藏高原腹地的西藏班戈天门 洞(Cai等, 2012)、湖北神农架三宝洞(Dong等, 2010)、 贵州董哥洞(D4)(Dykoski等, 2005)和贵州黑洞(Jiang 等, 2013)、印度东北部的Mawmluh洞(Berkelhammer 等, 2012)和阿曼Qunf洞 (Fleitmann等, 2003, 2007) 石 笋 $\delta^{18} \mathrm{O}$ 记录放在一起进行对比(图3). 高原天门洞在 WXB07-4顶部的生长时段, 由于高原垂直递减率的 放大作用(Cai等, 2012)以及高海拔地区对亚洲夏季风 的高灵敏度(Boos和Kuang, 2013)和较高的地形降水 量(Minder和Roe, 2005; Curio和Scherer, 2016), 天门洞 $\delta^{18} \mathrm{O}$ 值以 $4 \mathrm{a}$ 分辨率的大幅度波动分别记录了多次偏 负值的季风加强和多次偏重值的季风减弱阶段. 如果 考虑天门洞155a的平均定年误差, 所记录的主要季风 加强峰和减弱谷在万象记录中均能找到对应关系. 特 别是在天门记录的 5640 4920a BP的第3相内, 季风同 样处于减弱期且存在大幅度的波动 $\left(\delta^{18} \mathrm{O}\right.$ 值的波动幅 度达到 $3.88 \%$ ), 平均值整体偏正 $(-17.55 \%)$. 万象记录 中 5430a BP处的季风减弱谷在高海拔天门洞记录中 可能对应于 $5350 \mathrm{a} \mathrm{BP}$ 左右的 $\delta^{18} \mathrm{O}$ 值偏重谷; 此外, 天门 洞记录中分别在 $5060 \mathrm{a}$ BP、5170a BP左右出现2次大 幅度波动的季风减弱的 $\delta^{18} \mathrm{O}$ 值偏重谷(图3 $\mathrm{a}$ 和 $\mathrm{b}$ ). 高海 拔地区对亚洲夏季风降水的大幅度更敏感响应这种 事实从另外角度证实了万象洞石笋 $\delta^{18} \mathrm{O}$ 值的小幅度 波动也是季风降水波动的记录.

与万象洞第3相记录相对应的中国三宝洞(Dong 等, 2010)在 5530 4790a BP和董哥洞(D4)(Dykoski等, 2005)在 5560 4830a BP之间的石笋 $\delta^{18} \mathrm{O}$ 平均值(分别为 $-9.82 \%$ 和 $-8.47 \%$ )均偏重于研究时段的整体平均值(图 $3 \mathrm{c}$ 和d), 显示出季风的减弱期; 同时与黑洞5510 4850a $\mathrm{BP}$ 一样(图3e), 石笋 $\delta^{18} \mathrm{O}$ 值在这期间也显示大幅度的特 征变化. 但是, 除黑洞石笋 $\delta^{18} \mathrm{O}$ 值在这期间的极端偏重 值年代5440a BP与万象记录几乎相同外, 三宝洞、董 哥洞记录 $\delta^{18} \mathrm{O}$ 值极端偏重值却分别出现在5280a BP、 5180a BP左右, 与万象记录有一定的差异, 可能与测年 误差和样品分辨率(Rehfeld等, 2013)或者洞穴所在地 
对季风降水总强度差异性响应有关(Cheng等, 2012).

对于相当于万象洞记录的具有减弱季风特征的第 3相的印度Mawmluh洞(图3f, Berkelhammer等, 2012) 和阿曼 Qunf洞 (图 3g, Fleitmann等, 2003, 2007)石笋 $\delta^{18} \mathrm{O}$ 记录, 它们的时代跨度分别集中在5710 4880和 5510 4940a BP, 这期间的平均值 (分别为 $-6.07 \%$ 和 $-1.28 \%$ ) 均比整体平均值偏重. 其中 Mawmluh洞和 Qunf洞记录的大幅度季风减弱谷分别出现在 5350 和 5340a BP左右, 也与万象记录有100a左右的偏差. 同时 在Mawmluh洞记录的5570和5150a BP左右和Qunf洞记 录的 $5080 \mathrm{a}$ BP左右分别出现明显的季风减弱谷.

不同记录中的定年精度和数量、由生长速率造 成的采样分辨率和规则性均能影响它们之间的精确 对比(Rehfeld等, 2013). 对于本文研究的和对比的中国 天门洞(Cai等, 2012)、三宝洞(Dong等, 2010)、董哥洞 D4(Dykoski等, 2005)和黑洞(Jiang等, 2013)4个不同洞 穴中, 其定年误差(定年数量)/分辨率均具有大的差异, 分别为 $55 a(3) / 38 a 、 69 a(5) / 19 a$ 和 $29 a(4) / 21 a$; 同样地, 印度(Berkelhammer等, 2012)和阿曼Qunf洞(Fleitmann 等, 2003, 2007)除分辨率相对较高并接近万象记录外, 也有较大的定年误差, 分别为 $52 \mathrm{a}(4) / 6 \mathrm{a}$ 和 $99 \mathrm{a}(4) / 7 \mathrm{a}$. 如 此大的差异, 根据古气候网络规则(Rehfeld等, 2013)和 新近的亚洲季风区石笋全新世记录再循环网络(Donges 等, 2015)技术分析, 我们仍然能够确认第3相季风减弱 期间(图3)内不同幅度主要季风极端减弱谷的存在; 而 且所研究洞穴中如果扣除神农架 $-0.2 \% / 100 \mathrm{~m}$ (段福才 和孔兴功, 2011)和青藏高原 $-0.3 \% / 100 \mathrm{~m}$ (Yao等, 2009) 的降水氧同位素海拔效应, 从南到北几个石笋氧同位 素组成逐渐偏负(图1和3), 显示出热带季风雨带向大 陆的入侵(Winnick等, 2014).

\section{4 中全新世亚洲夏季风变迁的极端性与文化响 应}

万象石笋WXB07-4记录的在6420 4920a BP期间 亚洲夏季风强弱与之相联系的降水变化时间序列(图 3a), 恰好覆盖了位于亚州夏季风季风边缘或者前端地 区包括在河南、甘肃、青海、陕西、山西、内蒙古、 河北和辽宁部分地区在内的中晚新石器时代及青铜 器时代初期以农业起源和社会文化形成为特征的中 华文明快速发展时期(图3, 张之恒, 2009; Liu和Chen, 2012). 因此, 万象洞记录能够为这些文化的起源、发
展和变化提供明确的气候背景.

以广泛分布于中国黄土高原东-西部边缘及其周 边的仰韶文化为代表, 其中在以雨养旱地农业普通 粟类(Panicum miliaceum)(Crawford, 2009; Lu等, 2009; Barton等, 2009; Bettinger等, 2010; Zhao, 2011)为特征 的、发育在7000 6000a BP的仰韶早期黄河-渭河流域 半坡文化的衰落(Zhao, 2011; Peterson和Shelach, 2012), 主要对应于石笋WXB07-4记录的第1相亚洲夏季风降 水由多到少的逐渐过渡期以及第 2 相早期季风降水减 弱后的平稳波动期, 并连接着中仰韶文化前期向东 沿黄河中下游地区大规模的扩散、人口的增长( $\mathrm{Li}$ 等, 2009; Wagner等, 2013; Hosner等, 2016; Wang等, 2014; Li 等, 2015)、陆地使用面积的扩展(Yu等, 2016)与旱 地作物种类的增加使得㯟粟同时耕作(Barton等, 2009; Jin等, 2016)、和黄河下游大汶口文化在 6100a BP左 右(图3)的兴起. 直至5500a BP左右更耐旱重要作物 荞麦(Fagopyrum) 的出现以弥补㯟粟作物的降低( $\mathrm{Li}$ 等, 2009)、渭河河谷关中地区和河南西部以杨官寨遗址 为代表的仰韶中期庙底沟文化-黄河下游大汶口早期文 化的衰落(杨颖, 2013)并且人口出现小幅度的下降( $\mathrm{Li}$ 等, 2015). 这个时代恰好与WXB07-4记录的第3相中期 的季风降水极端降低期年代 5430a BP相一致, 这时黄 土高原整体出现干旱(Maher和Hu, 2006; Maher, 2008), 中国陕西关中盆地全新世古土壤在6000 5000a BP被 干旱和风尘沉积物所中断(Huang等, 2000; 杨颖, 2013), 高原和季风边缘区湖水位降低(Mischke和Zhang, 2010; An等, 2012; Xiao等, 2009). 与仰韶中期文化相比, 在黄 河下游地区分布的河南地区仰韶晚期文化和山东大 汶口中期文化的同时繁荣和演变、关中杨官寨遗址 和渭河上游大地湾、半坡四期仰韶晚期文化在 $4900 \mathrm{a}$ BP左右的最终消失(Barton等, 2009; 杨颖, 2013; Jin等, 2016), 几乎对应于万象记录的亚洲季风 $5430 \mathrm{a}$ BP极端 减弱谷及第 3 相 $5400 \mathrm{a}$ BP减弱事件后期亚洲夏季风降 水逐渐的增多、然后在 $4920 \mathrm{a}$ BP左右突然的大幅度减 少时期(图3).

同时孕育在6400a BP左右的我国辽河西部河谷地 带亚洲东北部季风边缘区红山早期文化的衰落也跟 随亚洲夏季风逐渐减弱所造成的降水逐渐减少(图3), 其中期文化在 5500a BP左右的衰落与万象石笋氧同位 素记录的亚洲夏季风5400a BP极端减弱事件前期季 风降水大幅度下降有关, 而红山晚期文化的建立特别 
是后期 5300a BP左右红山文明的形成直至 5000/4900a $\mathrm{BP}$ 左右的急剧衰落 (图3; 孙妙凝, 2016), 也与亚洲夏 季风 $5400 \mathrm{a}$ BP极端减弱事件后期季风降水逐步增加然 后突然减少有关. 而且长江中下游在 5400 和 $5300 \mathrm{a}$ BP 左右分别崛起的屈家岭文化和良渚文化(图3)也处于 与这个极端事件相联系的降水波动或者气候变异范 围之内. 对于 4920a BP左右季风在边缘区突然减弱(图 3a)和仰韶文化大规模崩溃后, 在黄河中下游地区向河 南龙山文化过渡期间庙底沟二期文化(简称庙二文化 或龙山早期)的建立、繁荣的大汶口晚期文化以及在 山东黄海平原山东龙山文化的兴起(Underhill等, 2008; Lanehart等, 2011), 可能连接着亚洲夏季风降水雨带的 向东南移动(Jiang和Liu, 2007). 亚洲夏季风4200a BP 突发性极端减弱的事件发生(图3b g) 可能导致黄河平 原龙山文化在 $3900 \mathrm{a} \mathrm{BP}$ 左右的崩溃或者演变、长江三 角洲新石器晚期代表性良渚文化在 $4300 \mathrm{a} \mathrm{BP}$ 左右的消 失或者过渡为后良渚即钱山漾和广富林文化(许鹏飞, 2015)、以及在屈家岭文化基础上发展起来的石家河 文化在 4000a BP左右的结束(Wu和Liu, 2004; Wang等, 2005; Chen和Wang, 2012; Innes等, 2014; Wu等, 2014a, 2014b; Wang等, 2016).

对于在黄河上游河谷地带5300a BP左右开始建立 的马家窑文化及其扩张(Dong等, 2013), 对应于西黄土 高原5430a BP左右季风降水极端减弱谷之后的逐渐 增加时期, 此时高原降水也突然开始增加(图3b), 可能 指示了人类在仰韶中期文化崩溃后为了水资源经过 近200a向黄河上游的迁徙, 并在高原东部合适位置的 定居并继续耕作仰韶时期的雨养旱地粟类农业(Zhao, 2011; Guedes等, 2014); 而且马家窑相的收缩恰好发生 在高原降水突然降低时期(图3b), 直至通过半山相-马 厂相的延续在亚洲季风极端减弱事件内 $4200 \mathrm{a}$ 左右最 干旱时消失和齐家文化的崛起(图3b、d、e、f和g). 同时高原人类在 $5200 \sim 3600 \mathrm{a} \mathrm{BP}$ 期间在 $2500 \mathrm{~m}$ asl左右 从事的主要雨养旱地粟类农业过渡到3600 2300a BP 期间在更高海拔(3400m asl左右)耕作雨养旱地大麦 农业(Chen等, 2015; Guedes等, 2015a, 2015b; Dong等, 2015), 可能与适应更高海拔地区对季风降水的高灵敏 度(Boos 和Kuang, 2013)和较高的地形降水量(Minder 和Roe, 2005; Curio和Scherer, 2016)所需的更耐寒大麦 农业耕作(Guedes, 2015; Guedes等, 2015a, 2015b)有关.

\section{4 结论}

高精度和高分辨率的黄土高原西部万象洞石笋 WXB07-4顶部 $\delta^{18} \mathrm{O}$ 值时间序列记录了从 $6420 \mathrm{a} \mathrm{BP}$ 到 4920a BP 期间的亚洲夏季风逐渐减弱趋势, 并显示3 相亚洲夏季风变迁特征, 包括由6420 6170a BP 具有 大幅度波动的季风强盛期和较高降水量的第 1 相过渡 到6170 5700a BP期间夏季风平稳波动的第 2 相，和在 5700 4920a BP期间出现的第3相亚洲夏季风突然出 现减弱期. 在第3相期间, 以 $5430 \mathrm{a}$ BP为年代突变点出 现亚洲夏季风极端减事件(5400a或5.4ka事件), 分别 经历了 $290 \mathrm{a}$ 的逐渐减弱和 $350 \mathrm{a}$ 的逐渐增强, 其在定年 误差范围之内与亚洲季风区其他洞穴石笋记录具有 等时性.

通过与考古资料的对比发现, 发生在7000 6000 a $\mathrm{BP}$ 的中国新石器晚期仰韶早期文化、黄河流域渭河 河谷半坡文化和西辽河流域红山文化早期的衰落发 生在亚洲夏季风降水由多到少的逐渐过渡期; 5400a $\mathrm{BP}$ 极端夏季风减弱事件发生导致的降水大幅度减少 可能与 5500a BP左右黄河流域渭河河谷关中地区杨官 寨遗址庙底沟文化、河南西部仰韶中期文化和下游 大汶口早期文化、辽河河谷西部红山中期文化的衰 落有关. 5400a BP事件后期(5430 4920a BP)与亚洲夏 季风相联系的降水小幅度逐渐增加和随后的剧烈减 少可能也与仰韶晚期文化和红山晚期文化的共同繁 荣和消失、渭河关中盆地以杨官寨遗址为代表的仰 韶晚期文化和上游地区大地湾第四期仰韶晚期文化 的消失、大汶口中期文化的开始与晚期文化的形成 以及龙山文化的兴起、长江中下游崛起的屈家岭文 化和良渚文化的相联系. 通过与高原石笋降水记录的 对比发现, 黄河上游河谷地带在 5300a BP左右兴起的 马家窑文化及其扩张也与 $5400 \mathrm{a} \mathrm{BP}$ 事件后期夏季风在 高海拔地区比在低海拔地区具有大幅度的降水增加 有关.

致谢 万象洞管理所对该项目提供了极大的洞穴考察 支持, 中国科学院南京地质古生物研究所同位素实验室 陈小明高级工程师完成了稳定同位素测试, 两名若名审 稿专家和鹿化暗教授提出了有价值的修改建议, 在此一 并表示感谢. 


\section{参考文献}

段福才, 孔兴功. 2011. 神农架石笋氧同位素的海拔效应. 中国岩 溶, 30: 101-104

韩建业. 2006. 晋西南豫西西部庙底沟二期-龙山时代文化的分期 与谱系. 考古学报, (2):179-204

李小强. 2013. 中国全新世气候和农业活动研究新进展. 中国科学: 地球科学, 43:1919-1928

桑文翠, 张德忠, 王晓锋, 白益军, 张平中, 吴秀平. 2013. 甘肃武都 万象洞方解石现代沉积控制因素分析. 第四纪研究, 33: 936-944 孙妙凝. 2016. 红山文化已进入文明时代一一访中国社会科学院考 古研究所研究员刘国祥. 《中国社会科学报》2016年3月18日 第 928 期

王守功. 2004. 山东龙山文化. 济南: 山东文艺出版社. 163

许鹏飞. 2015. 宁镇地区及环太湖地区新石器时代考古学文化研究. 博士学位论文. 长春: 吉林大学. 4-222

杨颖. 2013. 陕西杨官寨遗址仰韶文化中晚期气候环境记录及遗址 古人类对区域环境依存. 硕士学位论文. 西安: 西北大学. 3-56 张平中, 代志波, 安春雷, 李长久, 庞福顺, 龙路德, 马全龙, 贾继红. 2005. 甘肃南部又发现一处天然大溶洞一一黄爷洞. 第四纪研 究, 25: 795

张之恒. 2009. 中国新石器时代文化概述和中国各地区主要的新石 器时代文化. 见: 张之恒, 主编. 中国考古通论. 南京: 南京大学 出版社. 106-268

An C B, Feng Z, Tang L. 2004. Environmental change and cultural response between 8000 and $4000 \mathrm{cal}$. yr BP in the western Loess Plateau, northwest China. J Quat Sci, 19: 529-535

An Z, Colman S M, Zhou W, Li X, Brown E T, Jull A J T, Cai Y, Huang Y, Lu X, Chang H, Song Y, Sun Y, Xu H, Liu W, Jin Z, Liu X, Cheng P, Liu Y, Ai L, Li X, Liu X, Yan L, Shi Z, Wang X, Wu F, Qiang X, Dong J, Lu F, Xu X. 2012. Interplay between the Westerlies and Asian monsoon recorded in Lake Qinghai sediments since 32 ka. Sci Rep, 2: 619

Baker A J, Sodemann H, Baldini J U L, Breitenbach S F M, Johnson K R, van Hunen J, Zhang P. 2015. Seasonality of westerly moisture transport in the East Asian summer monsoon and its implications for interpreting precipitation $\delta^{18} \mathrm{O}$. J Geophys Res Atmos, 120: 5850-5862

Barton L, An C B. 2014. An evaluation of competing hypotheses for the early adoption of wheat in East Asia. World Archaeol, 46: 775-798

Barton L, Newsome S D, Chen F H, Wang H, Guilderson T P, Bettinger R L. 2009. Agricultural origins and the isotopic identity of domestication in northern China. Proc Natl Acad Sci USA, 106: 5523-5528

Berkelhammer M, Sinha A, Stott L, Cheng H, Pausata F S R, Yoshimura K. 2012. An abrupt shift in the Indian Monsoon 4000 years ago. Geophysical Monograph Series: Climate Landscapes and Civilization. Washington DC: American Geophysical Union. 75-87
Bettinger R L, Barton L, Morgan C. 2010. The origins of food production in north China: A different kind of agricultural revolution. Evol Anthropol, 19: 9-21

Boos W R, Kuang Z M. 2013. Sensitivity of the South Asian monsoon to elevated and non-elevated heating. Sci Rep, 3: 590-595

Budja M. 2015. Archaeology and rapid climate changes: From the collapse concept to a panarchy interpretative model. Doc Praeh, 42: 171-184

Butzer K W, Endfield G H. 2012. Critical perspectives on historical collapse. Proc Natl Acad Sci USA, 109: 3628-3631

Cai Y J, Zhang H W, Cheng H, An Z S, Lawrence Edwards R, Wang X F, Tan L C, Liang F Y, Wang J, Kelly M. 2012. The Holocene Indian monsoon variability over the southern Tibetan Plateau and its teleconnections. Earth Planet Sci Lett, 335-336: 135-144

Chen F H, Dong G H, Zhang D J, Liu X Y, Jia X, An C B, Ma M M, Xie Y W, Barton L, Ren X Y, Zhao Z J, Wu X H, Jones M K. 2015. Agriculture facilitated permanent human occupation of the Tibetan Plateau after 3600 B.P.. Science, 347: 248-250

Chen W, Wang W M. 2012. Middle-Late Holocene vegetation history and environment changes revealed by pollen analysis of a core at Qingdao of Shandong Province, East China. Quat Int, 254: 68-72

Cheng H, Edwards R L, Hoff J, Gallup C D, Richards D A, Asmerom Y. 2000. The half-lives of uranium-234 and thorium-230. Chem Geol, 169: $17-33$

Cheng H, Lawrence Edwards R, Shen C C, Polyak V J, Asmerom Y, Woodhead J, Hellstrom J, Wang Y, Kong X, Spötl C, Wang X, Calvin Alexander Jr. E. 2013. Improvements in ${ }^{230} \mathrm{Th}$ dating, ${ }^{230} \mathrm{Th}$ and ${ }^{234} \mathrm{U}$ half-life values, and U-Th isotopic measurements by multi-collector inductively coupled plasma mass spectrometry. Earth Planet Sci Lett, 371-372: 82-91

Cheng H, Fleitmann D, Edwards R L, Wang X, Cruz F W, Auler A S, Mangini A, Wang Y, Kong X, Burns S J, Matter A. 2009. Timing and structure of the $8.2 \mathrm{kyr}$ B.P. event inferred from ${ }^{18} \mathrm{O}$ records of stalagmites from China, Oman, and Brazil. Geology, 37: 1007-1010

Cheng H, Sinha A, Wang X, Cruz F W, Edwards R L. 2012. The Global Paleomonsoon as seen through speleothem records from Asia and the Americas. Clim Dyn, 39: 1045-1062

Cheng H, Edwards R L, Sinha A, Spötl C, Yi L, Chen S, Kelly M, Kathayat G, Wang X, Li X, Kong X, Wang Y, Nin Y, Zhang H. 2016. The Asian monsoon over the past 640000 years and ice age terminations. Nature, 534: 640-646

Chiang J C H, Fung I Y, Wu C H, Cai Y, Edman J P, Liu Y, Day J A, Bhattacharya T, Mondal Y, Labrousse C A. 2015. Role of seasonal transitions and westerly jets in East Asian paleoclimate. Quat Sci Rev, 108: $111-129$

Clarke J, Brooks N, Banning E B, Bar-Matthews M, Campbell S, Clare L, 
Cremaschi M, di Lernia S, Drake N, Gallinaro M, Manning S, Nicoll K, Philip G, Rosen S, Schoop U D, Tafuri M A, Weninger B, Zerboni A. 2016. Climatic changes and social transformations in the Near East and North Africa during the 'long' 4th millennium BC: A comparative study of environmental and archaeological evidence. Quat Sci Rev, 136: $96-121$

Crawford G W. 2009. Agricultural origins in North China pushed back to the Pleistocene-Holocene boundary. Proc Natl Acad Sci USA, 106: $7271-7272$

Cullen H M, deMenocal P B, Hemming S, Hemming G, Brown F H, Guilderson T, Sirocko F. 2000. Climate change and the collapse of the Akkadian empire: Evidence from the deep sea. Geology, 28: 379-382

Curio J, Scherer D. 2016. Seasonality and spatial variability of dynamic precipitation controls on the Tibetan Plateau. Earth Syst Dynam, 7: $767-782$

Dallmeyer A, Claussen M, Wang Y, Herzschuh U. 2013. Spatial variability of Holocene changes in the annual precipitation pattern: A model-data synthesis for the Asian monsoon region. Clim Dyn, 40: 2919-2936

Ding Y, Wang Z, Sun Y. 2008. Inter-decadal variation of the summer precipitation in East China and its association with decreasing Asian summer monsoon. Part I: Observed evidences. Int J Climatol, 28: 1139-1161

Dixit Y, Hodell D A, Petrie C A. 2014. Abrupt weakening of the summer monsoon in northwest India 4100 yr ago. Geology, 42: 339-342

Dodson J R, Li X, Zhou X, Zhao K, Sun N, Atahan P. 2013. Origin and spread of wheat in China. Quat Sci Rev, 72: 108-111

Dong G, Wang L, Cui Y, Elston R, Chen F. 2013. The spatiotemporal pattern of the Majiayao cultural evolution and its relation to climate change and variety of subsistence strategy during late Neolithic period in Gansu and Qinghai Provinces, northwest China. Quat Int, 316: $155-161$

Dong G H, Zhang D J, Liu X Y, Liu F G, Chen F H, Jones M. 2015. Response to Comment on "Agriculture facilitated permanent human occupation of the Tibetan Plateau after 3600 B.P.”. Science, 347: 872

Dong G H, Zhang D J, Liu X Y, Liu F G, Chen F H, Jones M. 2010. A high-resolution stalagmite record of the Holocene East Asian monsoon from Mt Shennongjia, central China. Holocene, 20: 257-264

Donges J F, Donner R V, Marwan N, Breitenbach S F M, Rehfeld K, Kurths J. 2015. Non-linear regime shifts in Holocene Asian monsoon variability: Potential impacts on cultural change and migratory patterns. Clim Past, 11: 709-741

Duan W, Ruan J, Luo W, Li T, Tian L, Zeng G, Zhang D, Bai Y, Li J, Tao T, Zhang P, Baker A, Tan M. 2016. The transfer of seasonal isotopic variability between precipitation and drip water at eight caves in the monsoon regions of China. Geochim Cosmochim Acta, 183: 250-266

Dykoski C A, Edwards R L, Cheng H, Yuan D, Cai Y, Zhang M, Lin Y, Qing J, An Z, Revenaugh J. 2005. A high-resolution, absolute-dated Holocene and deglacial Asian monsoon record from Dongge Cave, China. Earth Planet Sci Lett, 233: 71-86

Fleitmann D, Burns S J, Mangini A, Mudelsee M, Kramers J, Villa I, Neff U, Al-Subbary A A, Buettner A, Hippler D, Matter A. 2007. Holocene ITCZ and Indian monsoon dynamics recorded in stalagmites from Oman and Yemen (Socotra). Quat Sci Rev, 26: 170-188

Fleitmann D, Burns S J, Mudelsee M, Neff U, Kramers J, Mangini A, Matter A. 2003. Holocene forcing of the Indian monsoon recorded in a stalagmite from Southern Oman. Science, 300: 1737-1739

Fleitmann D, Mudelsee M, Burns S J, Bradley R S, Kramers J, Matter A. 2008. Evidence for a widespread climatic anomaly at around $9.2 \mathrm{ka}$ before present. Paleoceanography, 23: PA1102

Flohr P, Fleitmann D, Matthews R, Matthews W, Black S. 2016. Evidence of resilience to past climate change in Southwest Asia: Early farming communities and the 9.2 and $8.2 \mathrm{ka}$ events. Quat Sci Rev, 136: $23-39$

Guedes J A, Bocinsky R K, Butler E E. 2015a. Comment on “Agriculture facilitated permanent human occupation of the Tibetan Plateau after 3600 B.P. ". Science, 348: 872-872

Guedes J A, Lu H, Hein A M, Schmidt A H. 2015b. Early evidence for the use of wheat and barley as staple crops on the margins of the Tibetan Plateau. Proc Natl Acad Sci USA, 112: 5625-5630

Guedes J A, Lu H L, Li Y X, Spengler R N, Wu X L, Aldenderfer M S. 2014. Moving agriculture onto the Tibetan Plateau: The archaeobotanical evidence. Archaeol Anthropol Sci, 6: 255-269

Guedes J A. 2015. Rethinking the spread of agriculture to the Tibetan Plateau. Holocene, 25: 1498-1510

Hosner D, Wagner M, Tarasov P E, Chen X, Leipe C. 2016. Spatiotemporal distribution patterns of archaeological sites in China during the Neolithic and Bronze Age: An overview. Holocene, 26: 1576-1593

Huang C C, Zhou J, Pang J L, Han Y P, Hou C H. 2000. A regional aridity phase and its possible cultural impact during the Holocene Megathermal in the Guanzhong Basin, China. Holocene, 10: 135-142

Innes J B, Zong Y, Wang Z, Chen Z. 2014. Climatic and palaeoecological changes during the mid- to Late Holocene transition in eastern China: High-resolution pollen and non-pollen palynomorph analysis at Pingwang, Yangtze coastal lowlands. Quat Sci Rev, 99: 164-175

Jia X, Dong G, Li H, Brunson K, Chen F, Ma M, Wang H, An C, Zhang K. 2013. The development of agriculture and its impact on cultural expansion during the late Neolithic in the Western Loess Plateau, China. Holocene, 23: 85-92

Jiang W Y, Liu T S. 2007. Timing and spatial distribution of 
mid-Holocene drying over northern China: Response to a southeastward retreat of the East Asian Monsoon. J Geophys Res, 112: D24111

Jiang X, He Y, Shen C C, Li Z, Lin K. 2013. Replicated stalagmite-inferred centennial- to decadal-scale monsoon precipitation variability in southwest China since the mid Holocene. Holocene, 23: 841-849 Jin G Y, Wagner M, Tarasov P E, Wang F, Liu Y C. 2016. Archaeobotanical records of Middle and Late Neolithic agriculture from Shandong Province, East China, and a major change in regional subsistence during the Dawenkou Culture. Holocene, 26: 1605-1615

Johnson K R, Lynn Ingram B, Sharp W D, Zhang P. 2006. East Asian summer monsoon variability during Marine Isotope Stage 5 based on speleothem $\delta^{18} \mathrm{O}$ records from Wanxiang Cave, central China. Palaeogeogr Palaeoclimatol Palaeoecol, 236: 5-19

Kathayat G, Cheng H, Sinha A, Spötl C, Edwards R L, Zhang H, Li X, Yi L, Ning Y, Cai Y, Lui W L, Breitenbach S F M. 2016. Indian monsoon variability on millennial-orbital timescales. Sci Rep, 6: 24374

Kaufman D S, Axford Y L, Henderson A C G, McKay N P, Oswald W W, Saenger C, Anderson R S, Bailey H L, Clegg B, Gajewski K, Hu F S, Jones M C, Massa C, Routson C C, Werner A, Wooller M J, Yu Z. 2016. Holocene climate changes in eastern Beringia (NW North America) - A systematic review of multi-proxy evidence. Quat Sci Rev, 147: 312-339

Lanehart R E, Tykot R H, Underhill A P, Luan F, Yu H, Fang H, Fengshu C, Feinman G, Nicholas L. 2011. Dietary adaptation during the Longshan period in China: Stable isotope analyses at Liangchengzhen (southeastern Shandong). J Archaeol Sci, 38: 2171-2181

Li C, Lister D L, Li H, Xu Y, Cui Y, Bower M A, Jones M K, Zhou H. 2011. Ancient DNA analysis of desiccated wheat grains excavated from a Bronze Age cemetery in Xinjiang. J Archaeol Sci, 38: 115-119

Li H, An C, Fan W, Dong W, Zhao Y, Wang H. 2015. Population history and its relationship with climate change on the Chinese Loess Plateau during the past 10000 years. Holocene, 25: 1144-1152

Li X, Shang X, Dodson J, Zhou X. 2009. Holocene agriculture in the Guanzhong Basin in NW China indicated by pollen and charcoal evidence. Holocene, 19: 1213-1220

Li X, Dodson J, Zhou X, Zhang H, Masutomoto R. 2007. Early cultivated wheat and broadening of agriculture in Neolithic China. Holocene, 17: $555-560$

Liu L, Chen X C. 2012. The Archaeology of China: From the Late Paleolithic to the Early Bronze Age. Cambridge: Cambridge University Press. 400

Liu Y H, Henderson G M, Hu C Y, Mason A J, Charnley N, Johnson K R, Xie S C. 2013. Links between the East Asian monsoon and North Atlantic climate during the 8200 year event. Nat Geosci, 6: 117-120
Liu Y, Hu C. 2016. Quantification of southwest China rainfall during the 8.2 ka BP event with response to North Atlantic cooling. Clim Past, 12: $1583-1590$

Liu Z, Wen X, Brady E C, Otto-Bliesner B, Yu G, Lu H, Cheng H, Wang Y, Zheng W, Ding Y, Edwards R L, Cheng J, Liu W, Yang H. 2014. Chinese cave records and the East Asia summer monsoon. Quat Sci Rev, 83: 115-128

Lu H, Zhang J, Liu K, Wu N, Li Y, Zhou K, Ye M, Zhang T, Zhang H, Yang X, Shen L, Xu D, Li Q. 2009. Earliest domestication of common millet (Panicum miliaceum) in East Asia extended to 10000 years ago. Proc Natl Acad Sci USA, 106: 7367-7372

Maher B A, Hu M. 2006. A high-resolution record of Holocene rainfall variations from the western Chinese Loess Plateau: Antiphase behaviour of the African/Indian and East Asian summer monsoons. Holocene, 16: 309-319

Maher B A. 2008. Holocene variability of the East Asian summer monsoon from Chinese cave records: A re-assessment. Holocene, 18: $861-866$

Marshall M H, Lamb H F, Huws D, Davies S J, Bates R, Bloemendal J, Boyle J, Leng M J, Umer M, Bryant C. 2011. Late Pleistocene and Holocene drought events at Lake Tana, the source of the Blue Nile. glob Planet Change, 78: 147-161

Minder J R, Roe G H. 2005. Orographic precipitation. Annu Rev Earth Planet Sci, 33: 645-671

Mischke S, Zhang C. 2010. Holocene cold events on the Tibetan Plateau. Glob Planet Change, 72: 155-163

Mohtadi M, Prange M, Steinke S. 2016. Palaeoclimatic insights into forcing and response of monsoon rainfall. Nature, 533: 191-199

Orland I J, Edwards R L, Cheng H, Kozdon R, Cross M, Valley J W. 2015. Direct measurements of deglacial monsoon strength in a Chinese stalagmite. Geology, 43: 555-558

Peterson C E, Shelach G. 2012. Jiangzhai: Social and economic organization of a Middle Neolithic Chinese village. J Anthropol Archaeol, 31: $265-301$

Prasad V, Farooqui A, Sharma A, Phartiyal B, Chakraborty S, Bhandari S, Raj R, Singh A. 2014. Mid-late Holocene monsoonal variations from mainland Gujarat, India: A multi-proxy study for evaluating climate culture relationship. Palaeogeogr Palaeoclimatol Palaeoecol, 397: $38-51$

Rehfeld K, Marwan N, Breitenbach S F M, Kurths J. 2013. Late Holocene Asian summer monsoon dynamics from small but complex networks of paleoclimate data. Clim Dyn, 41: 3-19

Schiemann R, Lüthi D, Schär C. 2009. Seasonality and interannual variability of the westerly jet in the Tibetan Plateau region. J Clim, 22: 2940-2957

Shen C C, Lawrence Edwards R, Cheng H, Dorale J A, Thomas R B, 
Bradley Moran S, Weinstein S E, Edmonds H N. 2002. Uranium and thorium isotopic and concentration measurements by magnetic sector inductively coupled plasma mass spectrometry. Chem Geol, 185: $165-178$

Tan L C, Cai Y J, An Z S, Cheng H, Shen C C, Breitenbach S F M, Gao Y, Edwards R L, Zhang H W, Du Y J. 2015. A Chinese cave links climate change, social impacts, and human adaptation over the last 500 years. Sci Rep, 5: 12284

Tan L C, Cai Y J, An Z S, Edwards R L, Cheng H, Shen C C, Zhang H W. 2011. Centennial- to decadal-scale monsoon precipitation variability in the semi-humid region, northern China during the last 1860 years: Records from stalagmites in Huangye Cave. Holocene, 21: 287-296

Tan L, An Z, Huh C A, Cai Y, Shen C C, Shiau L J, Yan L, Cheng H, Edwards R L. 2014. Cyclic precipitation variation on the western Loess Plateau of China during the past four centuries. Sci Rep, 4: 6381

Underhill A P, Feinman G M, Nicholas L M, Fang H, Luan F, Yu H, Cai F. 2008. Changes in regional settlement patterns and the development of complex societies in southeastern Shandong, China. J Anthropol Archaeol, 27: 1-29

Wagner M, Tarasov P, Hosner D, Fleck A, Ehrich R, Chen X, Leipe C. 2013. Mapping of the spatial and temporal distribution of archaeological sites of northern China during the Neolithic and Bronze Age. Quat Int, 290-291: 344-357

Wang C, Lu H, Zhang J, Gu Z, He K. 2014. Prehistoric demographic fluctuations in China inferred from radiocarbon data and their linkage with climate change over the past 50000 years. Quat Sci Rev, 98: $45-59$

Wang J, Sun L, Chen L, Xu L, Wang Y, Wang X. 2016. The abrupt climate change near $4400 \mathrm{yr}$ BP on the cultural transition in Yuchisi, China and its global linkage. Sci Rep, 6: 27723

Wang Y J, Cheng H, Edwards R L, An Z S, Wu J Y, Shen C C, Dorale J A. 2001. A high-resolution absolute-dated Late Pleistocene monsoon record from Hulu Cave, China. Science, 294: 2345-2348

Wang Y, Cheng H, Edwards R L, He Y, Kong X, An Z, Wu J, Kelly M J, Dykoski C A, Li X. 2005. The Holocene Asian monsoon: Links to solar changes and north Atlantic climate. Science, 308: 854-857

Wang Y, Cheng H, Edwards R L, Kong X, Shao X, Chen S, Wu J, Jiang X, Wang X, An Z. 2008. Millennial- and orbital-scale changes in the East Asian monsoon over the past 224000years. Nature, 451: 1090-1093

Wanner H, Beer J, Bütikofer J, Crowley T J, Cubasch U, Flückiger J, Goosse H, Grosjean M, Joos F, Kaplan J O, Küttel M, Müller S A, Prentice I C, Solomina O, Stocker T F, Tarasov P, Wagner M, Widmann M. 2008. Mid- to Late Holocene climate change: An overview. Quat Sci Rev, 27: 1791-1828

Wanner H, Solomina O, Grosjean M, Ritz S P, Jetel M. 2011. Structure and origin of Holocene cold events. Quat Sci Rev, 30: 3109-3123

Webster P J, Magaña V O, Palmer T N, Shukla J, Tomas R A, Yanai M, Yasunari T. 1998. Monsoons: Processes, predictability, and the prospects for prediction. J Geophys Res, 103: 14451-14510

Welc F, Marks L. 2014. Climate change at the end of the Old Kingdom in Egypt around 4200 BP: New geoarchaeological evidence. Quate Int, 324: 124-133

Wu J Y, Wang Y J, Cheng H, Kong X G, Liu D B. 2012. Stable isotope and trace element investigation of two contemporaneous annuallylaminated stalagmites from northeastern China surrounding the "8.2 ka event”. Clim Past, 8: 1497-1507

Wu L, Zhu C, Zheng C, Ma C, Wang X, Li F, Li B, Li K. 2014a. Impact of Holocene climate change on the prehistoric cultures of Zhejiang region, East China. J Geogr Sci, 24: 669-688

Wu L, Zhu C, Zheng C, Li F, Wang X, Li L, Sun W. 2014b. Holocene environmental change and its impacts on human settlement in the Shanghai Area, East China. Catena, 114: 78-89

Wu Q, Zhao Z, Liu L, Granger D E, Wang H, Cohen D J, Wu X, Ye M, Bar-Yosef O, Lu B, Zhang J, Zhang P, Yuan D, Qi W, Cai L, Bai S. 2016. Outburst flood at 1920 BCE supports historicity of Chinas Great Flood and the Xia dynasty. Science, 353: 579-582

Wu W, Liu T. 2004. Possible role of the "Holocene Event 3" on the collapse of Neolithic Cultures around the Central Plain of China. Quat Int, 117: 153-166

Winnick M J, Chamberlain C P, Caves J K, Welker J M. 2014. Quantifying the isotopic 'continental effect'. Earth Planet Sci Lett, 406: $123-133$

Xiao J, Chang Z, Si B, Qin X, Itoh S, Lomtatidze Z. 2009. Partitioning of the grain-size components of Dali Lake core sediments: Evidence for lake-level changes during the Holocene. J Paleolimnol, 42: 249-260

Yao T D, Zhou H, Yang X X. 2009. Indian monsoon influences altitude effect of $\delta^{18} \mathrm{O}$ in precipitation/river water on the Tibetan Plateau. Chin Sci Bull, 54: 2724-2731

Yang X L, Zhang P Z, Chen F H, Huh C, Li H C, Cheng H, Johnson K R, Liu J H, An C L. 2007. Modern stalagmite oxygen isotopic composition and its implications of climatic change from a high-elevation cave in the eastern Qinghai-Tibet Plateau over the past 50 years. Chin Sci Bull, 52: 1238-1247

Yu Y, Wu H, Finke P A, Guo Z. 2016. Spatial and temporal changes of prehistoric human land use in the Wei River valley, northern China. Holocene, 26: 1788-1801

Yuan D, Cheng H, Edwards R L, Dykoski C A, Kelly M J, Zhang M, Qing J, Lin Y, Wang Y, Wu J, Dorale J A, An Z, Cai Y. 2004. Timing, duration, and transitions of the last interglacial Asian monsoon. Science, 304: 575-578

Zhang D Z, Zhang P Z, Sang W C, Cheng H, Wu X P, Yuan Y, Bai Y J, 
Wang J L, Jia J H. 2010. Implications of stalagmite density for past climate change: An example from stalagmite growth during the last deglaciation from Wanxiang Cave, western Loess Plateau. Chin Sci Bull, 55: 3936-3943

Zhang P, Cheng H, Edwards R L, Chen F, Wang Y, Yang X, Liu J, Tan M, Wang X, Liu J, An C, Dai Z, Zhou J, Zhang D, Jia J, Jin L, Johnson K R. 2008. A test of climate, sun, and culture relationships from an 1810-year Chinese cave record. Science, 322: 940-942

Zhang P, Johnson K R, Chen Y, Chen F, Ingram L, Zhang X, Zhang C, Wang S, Pang F, Long L. 2004. Modern systematics and environmental significance of stable isotopic variations in Wanxiang Cave, Wudu, Gansu, China. Chin Sci Bull, 49: 1649-1652

Zhao Z J. 2011. New Archaeobotanic data for the study of the origins of agriculture in China. Curr Anthropol, 52: S295-S306 